\title{
WEBS, ITERATION GROUPS, AND EQUIVALENT CHANGES IN PROBABILITIES
}

\author{
BY \\ JÁNOS ACZÉL (University of Waterloo), \\ GÜNTER ROTE (Technische Universität Graz), \\ AND \\ JENS SCHWAIGER (Karl-Franzens-Universität Graz)
}

\begin{abstract}
Yew-Kwang Ng [12] listed several "reasonable properties" for equivalent changes of probabilities and other proportions. He produced a family of functions satisfying all properties and asked whether there exist essentially different ones. We show that this is the case, by constructing uncountably many families of functions satisfying all properties. We show also that there are no other solutions. Our method establishes connections with webs (nets) and iteration groups. This may be of interest both in itself and for applications.
\end{abstract}

1. Introduction. In the paper [12], Yew-Kwang $\mathrm{Ng}$ deals with the question how equivalent changes for probabilities (or proportions and percentages) should be calculated. To show that this is not obvious, we quote a more recent example: an article in Newsweek ("Endangered Family", August 30, 1993, p. 26 or p. 38 in different editions) notes that between 1960 and 1989, the proportion of young white women giving birth out of wedlock rose from 9 to 22 percent "markedly faster" than it did for blacks. Had the rate for blacks-42 percent in 1960 - "kept pace with the white rate, it would have topped 100 percent by now. As things stand, it's 70 percent." (Our emphasis.) This makes one wonder about the author's standards for comparing changes in proportions. We see that different changes in proportions (and probabilities) can certainly not be compared by taking quotients; and taking differences does not work either, for the same reason: probabilities cannot go under 0 or above 1 (percentages not below $0 \%$ or above $100 \%$ ).

$\mathrm{Ng}$ lists several "reasonable properties" for equivalent changes or, equivalently, for the family of functions representing them. Then he gives explicitly one family of functions that have all these properties and asks whether this is essentially the only family satisfying these requirements and possibly an additional convexity property (although the

Received October 13, 1993.

1991 Mathematics Subject Classification. Primary 39B12; Secondary 14C21, 26A51, 39B22, 53A60, 60A99, 92G05.

Key words and phrases. Equivalent changes, equivalent differences, percentages, probability, geometric webs, iteration theory, convexity, difference measurement. 
word convexity is not used; the rest of the terminology there is also unconventional but recognizable and, with one exception to be stated below, clearly formulated).

In this paper we show how to construct, with the aid of functional equations, all families of functions satisfying the requirements in [12], both with and without convexity. One family is closely related to the normal (Gaussian) probability distribution. It turns out that there is a great variety of possibilities for defining equivalent changes. $\mathrm{Ng}$ suspected that one might be able to single out a "natural" or "best" solution. This is in fact not possible, at least on the basis of his explicit requirements alone. However, as will be discussed at the end, our characterization of all solutions leads to an interpretation of equivalent changes which allows us to select the functions defining equivalent changes in a problem-specific way.

We arrive at our results by showing that the graphs of the functions form a regular geometric web (net) and that by reparametrization this family of functions becomes an iteration group. This establishes connections to iteration theory which is of great importance nowadays for mathematics and its applications (think for instance of dynamical systems and fractals $[7,13])$.

In Sec. 2 we will discuss the basic properties listed in [12] and in Sec. 3 we will show that they define equivalence relations. In Sec. 4 we will prove a characterization theorem for the families of functions defined by ten of these properties. In Secs. 5 and 6 , we will deal with an additional symmetry property and an additional convexity property, respectively. We will discuss in which way these properties restrict the families of functions and prove corresponding characterization theorems. These two sections are complemented by several examples which demonstrate the diversity of possibilities of realizing the properties. The final section discusses the results. It turns out that our characterization theorems yield a very natural interpretation of the functions that furnish equivalent changes of probabilities.

2. The basic properties. The article [12] starts with the question (slightly rephrased): "Suppose that a probability (or other proportion) changes from $x_{1}$ to $x_{2}$. Given $y_{1}$, for what probability (or proportion) $y_{2}$ can one say that the change from $y_{1}$ to $y_{2}$ is equivalent to the change from $x_{1}$ to $x_{2}$ ?" Noting that $y_{2} / y_{1}=x_{2} / x_{1}$ or $y_{2}-y_{1}=x_{2}-x_{1}$ will not do because probabilities have to stay between 0 and 1 (and percentages between 0 and 100), the paper lists "reasonable properties" that the function

$$
y_{2}=F\left(x_{1}, y_{1}, x_{2}\right)
$$

should have. In what follows, we list these properties, in slightly different order, with the names given to them by Y.-K. Ng in quotation marks (except for Property 12 which we call convexity rather than monotonicity in $d y / d x)$. We modified $\mathrm{Ng}$ 's properties 1 , 5,8 , and 12 , and accordingly we write $1^{\prime}, 5^{\prime}, 8^{\prime}, 12^{\prime}$, and $12^{\prime \prime}$ for our corresponding properties.

The property 1 given in [12]: " $F$ exists for all values $x_{1}, y_{1}, x_{2}$ between and inclusive of 0 and 1", should be slightly changed because, as it stands, it contradicts the other properties, in particular, Properties 6, 3, 2, and 4. Indeed, in the solution given by Eq. (1) on p. 294 of [12] (formula (20) in the present paper), the function $F$ is not defined 
for $x_{1}=x_{2}=0 ; x_{1}=y_{1}=0 ; x_{2}=0, y_{1}=1 ; x_{1}=x_{2}=1 ; x_{1}=y_{1}=1$; and for $x_{2}=1, y_{1}=0$. (There is also a similar ambiguity and a slight misprint in Property 5 as formulated in [12].) Property 1 should be replaced by the following.

Property 1' ("Completeness"). $F\left(x_{1}, y_{1}, x_{2}\right)$ is defined for all values of $x_{1}, y_{1}$ strictly between 0 and 1 and for all values of $x_{2}$ between 0 and 1 ( 0 and 1 included).

Some combinations of 0 and 1 values would also be permissible for $x_{1}$ and $y_{1}$, but they are not needed.

Property 2 ("Uniqueness"). This just states that $F$ is a function.

PROPERTY 3 (Interchangeability). This establishes the possibility of exchanging $\left(x_{1}, y_{1}\right)$ and $\left(x_{2}, y_{2}\right)$ :

$$
F\left(x_{1}, y_{1}, x_{2}\right)=y_{2} \quad \text { implies } F\left(x_{2}, y_{2}, x_{1}\right)=y_{1}
$$

that is, $F\left(x_{2}, F\left(x_{1}, y_{1}, x_{2}\right), x_{1}\right)=y_{1}$.

From here on $x_{1}$ and $y_{1}$ are regarded as fixed while $x_{2}$ is variable, and we denote $F\left(x_{1}, y_{1}, x_{2}\right)$ by $f^{[i]}(x)=F\left(x_{1}, y_{1}, x\right)$. (This is denoted by $f^{i}(x)$ in [12].) It turns out that there is a whole family $\left\{f^{[i]}\right\}$ of such functions.

Property $5^{\prime}$ requires that the graph of exactly one $f^{[i]}$ go through each point of $] 0,1\left[^{2}\right.$, the interior of the unit square. (The word "interior" is missing in [12] but it is clear from Properties 6 and 7 that, for example, no graph goes through $(x, 0)$ for $x>0$.) This statement consists of two parts. The first is Ng's original Property 5, "Identity",

$$
F\left(x_{1}, y_{1}, x_{1}\right)=y_{1}
$$

whose meaning is obvious: If $x$ remains unchanged, then also $y$ has to remain unchanged. It implies that at least one function $f^{[i]}$ goes through the point $\left(x_{1}, y_{1}\right)$. The statement that at most one function goes through this point is contained in [12] only as an afterthought to Property 7. In terms of $F$ it can be phrased as follows. Every function $f^{[j]}(x)=F\left(x_{2}, y_{2}, x\right)$ whose graph also goes through the point $\left(x_{1}, y_{1}\right)$ must coincide with the above function $f^{[i]}(x)=F\left(x_{1}, y_{1}, x\right)$ :

$$
F\left(x_{2}, y_{2}, x_{1}\right)=y_{1} \quad \text { and } \quad F\left(x_{2}, y_{2}, x_{3}\right)=y_{3} \quad \text { imply } \quad F\left(x_{1}, y_{1}, x_{3}\right)=y_{3}
$$

The interpretation of this is natural: If a change of $x$ from $x_{2}$ to $x_{1}$ is equivalent to a change of $y$ from $y_{2}$ to $y_{1}$ and another change from $x_{2}$ to $x_{3}$ is equivalent to a change from $y_{2}$ to $y_{3}$, then the direct change from $x_{1}$ to $x_{3}$ should be equivalent to a change from $y_{1}$ to $y_{3}$. We note that Property 3 follows from this interpretation of Property $5^{\prime}$. Indeed, choose in (4) $x_{3}=x_{2}, y_{3}=y_{2}$ in order to get

$$
F\left(x_{2}, y_{2}, x_{1}\right)=y_{1} \quad \text { and } \quad F\left(x_{2}, y_{2}, x_{2}\right)=y_{2} \quad \text { imply } \quad F\left(x_{1}, y_{1}, x_{2}\right)=y_{2}
$$

By (3) the second equality is always fulfilled, and by renaming $x_{1}, y_{1}$ to $x_{2}, y_{2}$ and vice versa we get $(2)$.

A consequence of Property $5^{\prime}$ is that there are uncountably many functions $f^{[i]}$ in the family. (Their cardinality is the same as that of the set $\mathbb{R}$ of real numbers.) 
Property 4 (which is called "Parity" in [12]). This establishes that the family $\left\{f^{[i]}\right\}$ contains the identity function given by $y=x$ or, in terms of $F$, by $F\left(x_{1}, x_{1}, x_{2}\right)=x_{2}$ : If $y_{1}=x_{1}$, i.e., $y$ starts at the same value as $x$, then we should have $y=x$ for all $x$ to effect a corresponding change.

Property 7 (Monotonicity). All functions $f^{[i]}$ are strictly increasing on the closed interval $[0,1]$.

Property 6 ("Limitation"). This postulates that $f^{[i]}(0)=0, f^{[i]}(1)=1$ for all $f^{[i]}$, that is, the graph of each $f^{[i]}$ goes through $(0,0)$ and $(1,1)$. In terms of $F$ this means that $F\left(x_{1}, y_{1}, 0\right)=0$ and $F\left(x_{1}, y_{1}, 1\right)=1$ for all $x_{1}, y_{1}$ in the open interval $] 0,1$ [. In other words, the (only) change of probability from $\left.y_{1} \in\right] 0,1[$ that is equivalent to a change of probability from $\left.x_{1} \in\right] 0,1$ [ to 0 or 1 is the change from $y_{1}$ to 0 or 1 , respectively.

Property 8 in [12] demands that all $f^{[i]}$ be continuously differentiable on $[0,1]$. We will need only continuity (in fact, we will derive differentiability from the additional convexity property in Sec. 6; see Theorem 3):

Property $8^{\prime}$ (Continuity). All functions $f^{[i]}$ (or all $\left.F\left(x_{1}, y_{1}, \cdot\right)\right)$ are continuous on $[0,1]$.

Property 9 is a certain symmetry property, which we will discuss in Sec. 5 .

Property 10 ("Anonymity"). This postulates that the inverse function of each $f^{[i]}$, which exists by Properties 6, 7, and $8^{\prime}$, also belong to the family. This means in terms of $F$ that $F\left(y_{1}, x_{1}, \cdot\right)$ is the inverse function of $F\left(x_{1}, y_{1}, \cdot\right)$, that is,

$$
F\left(x_{1}, y_{1}, x_{2}\right)=y_{2} \text { implies } F\left(y_{1}, x_{1}, y_{2}\right)=x_{2},
$$

or $F\left(y_{1}, x_{1}, F\left(x_{1}, y_{1}, x_{2}\right)\right)=x_{2}$, a property similar to Property 3 , this time establishing that $x_{1}$ and $x_{2}$ can be exchanged for $y_{1}$ and $y_{2}$. If the change from $x_{1}$ to $x_{2}$ is equivalent to the change from $y_{1}$ to $y_{2}$ then the change from $y_{1}$ to $y_{2}$ is clearly equivalent to that from $x_{1}$ to $x_{2}$. (It should not matter whether we call the first variable $x$ or $y$; this explains the name anonymity.)

PROPERTY 11 (Transitivity). This requires the following: "If the change in the variable $x$ from $x_{1}$ to $x_{2}$ is equivalent to the change in $y$ from $y_{1}$ to $y_{2}$ and to the change in $z$ from $z_{1}$ to $z_{2}$ then the change in $y$ from $y_{1}$ to $y_{2}$ is also equivalent to the change in $z$ from $z_{1}$ to $z_{2}$." The geometric interpretation is interesting: "If $\left(x_{1}, y_{1}\right)$ and $\left(x_{2}, y_{2}\right)$ lie on the same graph and $\left(x_{1}, z_{1}\right)$ and $\left(x_{2}, z_{2}\right)$ lie on the same graph (possibly different from the previous graph) then also $\left(z_{1}, y_{1}\right)$ and $\left(z_{2}, y_{2}\right)$ lie on the same graph (possibly different from the previous two)." See Fig. 1.

We have already seen above that Property 3 is a consequence of Property $5^{\prime}$. We will now show that Property 11, in conjunction with Properties 4 and $5^{\prime}$, is even more powerful: it implies that every $f^{[i]}$ is a one-to-one mapping (bijection). From this we will be able to show that several properties can be omitted.

Let us set $x_{1}=y_{1}$ and $x_{2}=y_{2}$ in Property 11. Then we get the following special case: "If $\left(x_{1}, x_{1}\right)$ and $\left(x_{2}, x_{2}\right)$ lie on the same graph (which they always do, by Property $4)$ and $\left(x_{1}, z_{1}\right)$ and $\left(x_{2}, z_{2}\right)$ lie on the same graph then also $\left(z_{1}, x_{1}\right)$ and $\left(z_{2}, x_{2}\right)$ lie on the same graph." Thus we observe that the set of graphs of the functions $f^{[i]}$ is invariant under the exchange of coordinates; in other words, they must lie symmetric with respect to the $45^{\circ}$ line $y=x$. This implies that the functions are bijections, as is formulated in the following lemma. 


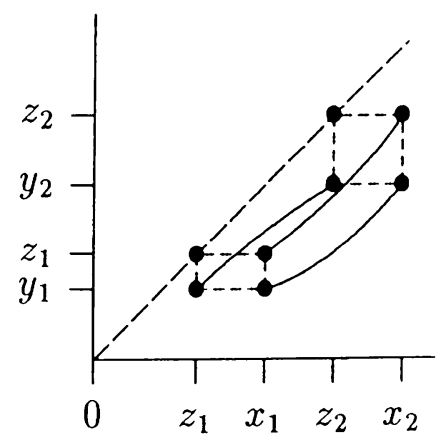

FIG. 1. The transitivity property (Reidemeister condition)

Lemma. Properties $1^{\prime}, 2,4,5^{\prime}$, and 11 imply that all functions $f^{[i]}$ are bijections of the interval ]0, 1[ onto itself.

Proof. To show that the functions are injective, assume that the points

$\left(x_{1}, z\right)$ and $\left(x_{2}, z\right)$ with $x_{1} \neq x_{2}$ lie on the same curve. By the above observation, the points $\left(z, x_{1}\right)$ and $\left(z, x_{2}\right)$ lie also on the same curve, contradicting the fact that the curve is the graph of a function (Properties $1^{\prime}$ and 2).

Surjectivity means that the equation $z_{2}=f^{[i]}\left(x_{2}\right)$ has a solution $x_{2}$ for all functions $f^{[i]}$ and all $\left.z_{2} \in\right] 0,1\left[\right.$. Set $x_{1}=1 / 2$ (or any other fixed value in $] 0,1\left[\right.$ ), $z_{1}=f^{[i]}\left(x_{1}\right.$ ), and consider the function $f^{[j]}$ going through the point $\left(z_{1}, x_{1}\right)$, which exists by Property $5^{\prime}$. We show that $x_{2}=f^{[j]}\left(z_{2}\right)$ is the required solution. Indeed, the point $\left(z_{2}, x_{2}\right)$ lies on the same curve as $\left(z_{1}, x_{1}\right)$. Hence, by the above observation, the point $\left(x_{2}, z_{2}\right)$ lies on the same curve as $\left(x_{1}, z_{1}\right)$. By Property $5^{\prime}$, this curve is unique, namely the graph of $f^{[i]}$. Thus we get $z_{2}=f^{[i]}\left(x_{2}\right)$ and we are done.

Property 10 follows directly from the above observation. A one-to-one mapping $f^{[i]}$ of an interval onto an interval is continuous if and only if it is strictly monotonic. So Properties 7 and $8^{\prime}$ are equivalent. (Monotonically decreasing continuous functions can be excluded since the graph of such a function would cross the graph of the identity function, contradicting Property $5^{\prime}$.) Properties 7 and $8^{\prime}$ together clearly imply Property 6 .

To summarize, we have seen that Properties 3,6, 10, and 7 or $8^{\prime}$ can be omitted, and it is sufficient to assume Properties $1^{\prime}, 2,4,5^{\prime}, 11$, and one of 7 and $8^{\prime}$.

3. Equivalence relations between pairs of probabilities. Before we investigate further consequences of our properties, we step back and look at them from a different point of view. The function $F$, with

$$
y_{2}=F\left(x_{1}, y_{1}, x_{2}\right)
$$

establishes a quaternary relation between $x_{1}, y_{1}, x_{2}$, and $y_{2}$.

However, the very wording of the interpretation that we have given to this relation, "the change from $y_{1}$ to $y_{2}$ is equivalent to the change from $x_{1}$ to $x_{2}$ ", suggests that we 
should view it as a binary relation between the pairs $\left(x_{1}, x_{2}\right)$ and $\left(y_{1}, y_{2}\right)$ and, moreover, that it should be an equivalence relation. Using the more suggestive notation

$$
\left(x_{1} \curvearrowright x_{2}\right) \sim\left(y_{1} \curvearrowright y_{2}\right)
$$

instead of (1), we find the properties of an equivalence relation in our properties: Reflexivity- $\left(x_{1} \curvearrowright x_{2}\right) \sim\left(x_{1} \curvearrowright x_{2}\right)$-is just a translation of $x_{2}=F\left(x_{1}, x_{1}, x_{2}\right)$, which is Property 4. Symmetry- $\left(x_{1} \curvearrowright x_{2}\right) \sim\left(y_{1} \curvearrowright y_{2}\right)$ implies $\left(y_{1} \curvearrowright y_{2}\right) \sim\left(x_{1} \curvearrowright x_{2}\right)$-is found as Property 10 about the inverse function. Finally, transitivity- $\left(x_{1} \curvearrowright x_{2}\right) \sim$ $\left(y_{1} \curvearrowright y_{2}\right)$ and $\left(x_{1} \curvearrowright x_{2}\right) \sim\left(z_{1} \curvearrowright z_{2}\right)$ together imply $\left(y_{1} \curvearrowright y_{2}\right) \sim\left(z_{1} \curvearrowright z_{2}\right)$-was already identified as a transitivity property, namely as Property 11.

On the other hand, we may view (1) also as a relation between the pairs $\left(x_{1}, y_{1}\right)$ and $\left(x_{2}, y_{2}\right)$. (In order to get on equal footing, we here restrict also $x_{2}$ and $y_{2}$ to $] 0,1[$.) The statement

$$
\left(x_{1}, y_{1}\right) \cdot \sim\left(x_{2}, y_{2}\right)
$$

may be given the interpretation: "The pair $\left(x_{1}, y_{1}\right)$ of initial values for $x$ and $y$ corresponds to the pair $\left(x_{2}, y_{2}\right)$ of final values for these quantities." Again, the wording suggests that this is an equivalence relation, which is indeed the case: Reflexivity$\left(x_{1}, y_{1}\right) \dot{\sim}\left(x_{1}, y_{1}\right)$--is the existence part of Property $5^{\prime}$; symmetry- $-\left(x_{1}, y_{1}\right) \dot{\sim}\left(x_{2}, y_{2}\right)$ implies $\left(x_{2}, y_{2}\right) \dot{\sim}\left(x_{1}, y_{1}\right)$-is Property 3 ; finally, transitivity- $\left(x_{2}, y_{2}\right) \dot{\sim}\left(x_{1}, y_{1}\right)$ and $\left(x_{2}, y_{2}\right)$ $\dot{\sim}\left(x_{3}, y_{3}\right)$ imply $\left(x_{1}, y_{1}\right) \dot{\sim}\left(x_{3}, y_{3}\right)$-is the uniqueness part of Property $5^{\prime}$.

Note that we formulated transitivity in such a way that, in conjunction with reflexivity, it should imply symmetry. The usual formulation of transitivity, $a \sim b$ and $b \sim c$ implies $a \sim c$, can then be directly obtained by symmetry.

All properties mentioned so far in this section $\left(3,4,5^{\prime}, 10\right.$, and 11) are purely algebraic in the sense that they assume nothing about the set of values that the quantities $x, y, z$ can take. Properties 1 and 2 are basic in the sense that they establish the objects that one deals with. The only further properties in Sec. 2 that have not been identified as properties of equivalence relations are 6,7 , and $8^{\prime}$. These have to do with the particular form of the functions and are of a different type. Properties $12^{\prime}$ and $12^{\prime \prime}$ will be further requirements of this type. Only Property 9, which will be introduced in Sec. 5, is of an algebraic nature again. It uses the structure of the involutory mapping $x \mapsto 1-x$ (complementation) on the underlying set.

\section{Geometric webs; iteration groups; the first characterization theorem.}

We now continue to explore the consequences of the properties and, in particular, we take a closer look at Property 11.

By Properties 6, 7, and $8^{\prime}$, the functions $f^{[i]}$ are continuous strictly increasing bijections of the interval ]0,1[ onto itself. By Property $5^{\prime}$, the graphs of these functions and the $x=$ constant, $y=$ constant lines form a geometric web or net (see $[1,2,5,6]$ ), that is, three families of continuous curves on a subset of the plane, in this case on $] 0,1\left[^{2}\right.$, such that each point of the subset lies on exactly one curve of each family and two curves of different families always meet in exactly one point. Property 11 is exactly the Reidemeister condition (see Fig. 1 and $[1,2,5]$ ) for three curves of the same family together with the one $y=x$, which belongs, by Property 4 , also to the family. 
As shown in [1] (see also [2]), if the Reidemeister condition is satisfied with one fixed curve of the third family (the three other curves of the third family and the "curves" of the other two families are arbitrary within the constraints of the condition) then it is always satisfied. Moreover, there exist continuous and strictly monotonic bijections (homeomorphisms) $\varphi, \psi, \Psi:] 0,1[\rightarrow \mathbb{R}$ such that the "contour lines" $z=$ constant of the equation

$$
\Psi(z)=\psi(x)+\varphi(y)
$$

are the curves of the third family, while, as mentioned above, the first two families consist of the vertical and horizontal lines $x=$ constant and $y=$ constant, respectively. So the curves of the third family are given by

$$
y=\varphi^{-1}(\Psi(z)-\psi(x))
$$

This has to be the same as $f^{[i]}(x)$ since the curves of the third family are the graphs of the functions $f^{[i]}$, and the choice of the value of $z$, or $\tau=\Psi(z)$ for that matter, picks an individual $f^{[i]}$ from the family of functions $\left\{f^{[i]}\right\}$. We will thus reparameterize and introduce a meaningful parameter $\tau$ in place of $i$ to which no particular meaning was attached, writing $f^{(\tau)}=f^{[i]}$ by definition. So we have

$$
y=f^{(\tau)}(x)=\varphi^{-1}(\tau-\psi(x))
$$

Since $\psi:] 0,1[\rightarrow \mathbb{R}$ is a bijection, $\tau=\psi(z)$ assumes every real value. Moreover, the identity function $y=x$ belongs, by Property 4 , to $\left\{f^{[i]}\right\}$ and so now to $\left\{f^{(\tau)}\right\}$. Let the parameter belonging to it be $\tau_{0}$, that is,

$$
\varphi^{-1}\left(\tau_{0}-\psi(x)\right)=x
$$

thus, $\psi(x)=\tau_{0}-\varphi(x)$ and

$$
f^{(\tau)}(x)=\varphi^{-1}\left(\tau-\tau_{0}+\varphi(x)\right)
$$

As a slight second reparametrization we introduce the parameter $t=\tau-\tau_{0}$ and write $f^{t}=f^{(\tau)}$. With $\tau$, also $t=\tau-\tau_{0}$ runs through the entire set of real numbers. Thus, our family of functions $\left\{f^{t}\right\}$ is given by

$$
\left.f^{t}(x)=\varphi^{-1}(\varphi(x)+t) \quad \text { for all } x \in\right] 0,1[; t \in \mathbb{R} \text {. }
$$

As for the identity function, it clearly belongs to the parameter value $t=0$ :

$$
f^{0}(x)=x
$$

The functions $f^{t}$ given by (5) satisfy

$$
f^{t}\left(f^{u}(x)\right)=\varphi^{-1}\left(t+\varphi\left[f^{u}(x)\right]\right)=\varphi^{-1}(t+u+\varphi(x))=f^{t+u}(x) .
$$


Thus, they form an iteration semigroup or, since the identity function is included, an iteration monoid. But, by Property 10, also the inverse function of any $f^{t}$ belongs to this monoid, let us call it $f^{t^{\prime}}$. By the definition of inverse functions we have

$$
x=f^{t^{\prime}}\left(f^{t}(x)\right)=\varphi^{-1}\left(t+t^{\prime}+\varphi(x)\right),
$$

that is, $\varphi(x)=t+t^{\prime}+\varphi(x)$ for all $x$, and $t^{\prime}=-t$. Therefore, $f^{-t}$ is the inverse of $f^{t}$ and $\left\{f^{t}\right\}$ is an iteration group. Concerning iteration groups, connected to webs or otherwise, see, for example, $[2,3,4,11]$.

If we want the graph of $f^{t}$ to pass through $\left(x_{1}, y_{1}\right)$, then

$$
y_{1}=f^{t}\left(x_{1}\right)=\varphi^{-1}\left(t+\varphi\left(x_{1}\right)\right)
$$

so, $t=\varphi\left(y_{1}\right)-\varphi\left(x_{1}\right)$ and $y=f^{t}(x)=\varphi^{-1}\left(\varphi\left(y_{1}\right)-\varphi\left(x_{1}\right)+\varphi(x)\right)$. Therefore,

$$
y_{2}=F\left(x_{1}, y_{1}, x_{2}\right)=\varphi^{-1}\left(\varphi\left(y_{1}\right)-\varphi\left(x_{1}\right)+\varphi\left(x_{2}\right)\right)
$$

describes the function whose graph goes through $\left(x_{1}, y_{1}\right)$, and (1) can be written as

$$
\varphi\left(y_{2}\right)-\varphi\left(y_{1}\right)=\varphi\left(x_{2}\right)-\varphi\left(x_{1}\right) \text {. }
$$

Thus, it turns out that

$$
F(x, y, z)=F(x, z, y)
$$

In (5) and (6) we had $\varphi$ continuous and strictly monotonic. Without loss of generality we may suppose that $\varphi$ is strictly increasing, by changing $t$ to $-t$ if necessary.

Until now we were on the open square $] 0,1\left[^{2}\right.$. Adding the two points $(0,0)$ and $(1,1)$ according to Property 6 we would give, if we wanted to keep the representations (5) and (6),

$$
0=f^{t}(0)=\varphi^{-1}(t+\varphi(0)) \quad \text { and } \quad 1=f^{t}(1)=\varphi^{-1}(t+\varphi(1))
$$

that is,

$$
\varphi(0)=t+\varphi(0) \quad \text { and } \quad \varphi(1)=t+\varphi(1), \quad \text { for all } t \in \mathbb{R} .
$$

Thus, $\varphi(0)$ and $\varphi(1)$ cannot be finite real numbers, but we can define them as $-\infty$ and $+\infty$ if we want to keep (5), (6), and the definitions as stated in Property 6 :

$$
\varphi(0)=-\infty, \varphi(1)=+\infty, \quad \text { and accordingly, } \quad \varphi^{-1}(-\infty)=0, \varphi^{-1}(+\infty)=1 .
$$

This definition keeps $\varphi$ and $\varphi^{-1}$ increasing and even continuous in the following sense. Since $\varphi$ is an increasing bijection of $] 0,1[$ onto $\mathbb{R}$, we have

$$
\lim _{x \rightarrow 0} \varphi(x)=-\infty, \quad \lim _{x \rightarrow 1} \varphi(x)=\infty, \quad \text { and } \quad \lim _{s \rightarrow-\infty} \varphi^{-1}(s)=0, \quad \lim _{s \rightarrow \infty} \varphi^{-1}(s)=1 .
$$

It is easy to check that the functions given by (5) and (6), with the extension (8), have all the properties $1^{\prime}, 2-4,5^{\prime}, 6,7,8^{\prime}, 10$, and 11 . Indeed, we have just forced Property 6 to hold:

$$
\begin{aligned}
& F\left(x_{1}, y_{1}, 0\right)=\varphi^{-1}\left(\varphi\left(y_{1}\right)-\varphi\left(x_{1}\right)-\infty\right)=\varphi^{-1}(-\infty)=0 \\
& F\left(x_{1}, y_{1}, 1\right)=\varphi^{-1}\left(\varphi\left(y_{1}\right)-\varphi\left(x_{1}\right)+\infty\right)=\varphi^{-1}(+\infty)=1
\end{aligned}
$$


Property $1^{\prime}$ is obvious, (6) defines $F$ for all $\left.x_{1}, y_{1}, x_{2} \in\right] 0,1\left[\right.$, and the cases $x_{2}=0$ and $x_{2}=1$ have just been discussed. Property 2 is self-evident. We check Property 3 by substituting (6) into it:

$$
\begin{aligned}
F\left(x_{2}, F\left(x_{1}, y_{1}, x_{2}\right), x_{1}\right) & =\varphi^{-1}\left(\varphi\left(F\left(x_{1}, y_{1}, x_{2}\right)\right)-\varphi\left(x_{2}\right)+\varphi\left(x_{1}\right)\right) \\
& =\varphi^{-1}\left(\varphi\left(y_{1}\right)-\varphi\left(x_{1}\right)+\varphi\left(x_{2}\right)-\varphi\left(x_{2}\right)+\varphi\left(x_{1}\right)\right) \\
& =\varphi^{-1}\left(\varphi\left(y_{1}\right)\right)=y_{1} .
\end{aligned}
$$

Properties 4 and 10 we have checked already: $f^{0}$ is the identity function, $f^{-t}$ is the inverse function of $f^{t}$. Property $5^{\prime}$ is satisfied because the graph of $f^{\varphi\left(y_{1}\right)-\varphi\left(x_{1}\right)}$ (and of no other $\left.f^{t}\right)$ goes through $\left(x_{1}, y_{1}\right)$, by the choice of $t$ in the derivation of (6) above:

$$
f^{\varphi\left(y_{1}\right)-\varphi\left(x_{1}\right)}\left(x_{1}\right)=\varphi^{-1}\left(\varphi\left(x_{1}\right)+\varphi\left(y_{1}\right)-\varphi\left(x_{1}\right)\right)=y_{1} .
$$

Properties 7 and $8^{\prime}$ are obvious because $\varphi$ is continuous and strictly monotonic.

Finally, we check Property 11. Do the equations

$$
f^{t}\left(x_{1}\right)=y_{1}, f^{t}\left(x_{2}\right)=y_{2}, \quad \text { and } \quad f^{u}\left(x_{1}\right)=z_{1}, f^{u}\left(x_{2}\right)=z_{2}
$$

that is,

$$
t+\varphi\left(x_{1}\right)=\varphi\left(y_{1}\right), t+\varphi\left(x_{2}\right)=\varphi\left(y_{2}\right), \quad \text { and } \quad u+\varphi\left(x_{1}\right)=\varphi\left(z_{1}\right), u+\varphi\left(x_{2}\right)=\varphi\left(z_{2}\right)
$$

indeed imply that there exists a $v$ such that $f^{v}\left(z_{1}\right)=y_{1}, f^{v}\left(z_{2}\right)=y_{2}$, that is,

$$
v+\varphi\left(z_{1}\right)=\varphi\left(y_{1}\right) \quad \text { and } \quad v+\varphi\left(z_{2}\right)=\varphi\left(y_{2}\right) ?
$$

Yes, and $v=t-u$ does the job because from (9)

$$
\varphi\left(y_{1}\right)-\varphi\left(z_{1}\right)=t-u=\varphi\left(y_{2}\right)-\varphi\left(z_{2}\right)
$$

This concludes the proof of the following theorem.

THEOREM 1. The general families of functions satisfying the requirements $1^{\prime}, 2,3,4$, $5^{\prime}, 6,7,8^{\prime}, 10$, and 11 are given by (5) and (6), where $\varphi$ is an arbitrary continuous and strictly increasing bijective mapping from $] 0,1[$ onto $\mathbb{R}$, extended by (8).

We show now that for a given family of functions, $\varphi$ is unique up to positive affine transformations $\varphi(x) \mapsto a \cdot \varphi(x)+b$ with $a>0$. Suppose that two functions $\varphi$ and $\tilde{\varphi}$ give rise to the same family of functions $\left\{f^{t} \mid t \in \mathbb{R}\right\}=\left\{g^{u} \mid u \in \mathbb{R}\right\}$ with

$$
f^{t}(x)=\varphi^{-1}(\varphi(x)+t)
$$

and

$$
g^{u}(x)=\tilde{\varphi}^{-1}(\tilde{\varphi}(x)+u) .
$$

The two parametrizations must be related by some bijective function $\lambda: \mathbb{R} \rightarrow \mathbb{R}$ :

$$
g^{\lambda(t)}(x)=f^{t}(x)
$$


which is equivalent to

$$
\left.\tilde{\varphi}^{-1}(\tilde{\varphi}(x)+\lambda(t))=\varphi^{-1}(\varphi(x)+t) \quad \text { for all } x \in\right] 0,1[\text { and } t \in \mathbb{R} .
$$

By setting $\rho:=\tilde{\varphi} \circ \varphi^{-1}$ and $x=\varphi^{-1}(u)$ we obtain $\tilde{\varphi}(x)=\tilde{\varphi}\left(\varphi^{-1}(u)\right)=\rho(u)$ and

$$
\rho(u+t)=\tilde{\varphi}\left(\varphi^{-1}(\varphi(x)+t)\right)=\tilde{\varphi}\left(\tilde{\varphi}^{-1}(\tilde{\varphi}(x)+\lambda(t))\right)=\rho(u)+\lambda(t),
$$

for all $u, t \in \mathbb{R}$. The functional equation $\rho(u+t)=\rho(u)+\lambda(t)$ is a Pexider equation. It implies that $\rho(u+t)-\rho(u)$ depends only on $t$ and, since $\rho$ is continuous, the solution can only be of the form

$$
\rho(u)=a u+b
$$

for some constants $a$ and $b$; see [10]. Thus we get

$$
\tilde{\varphi}(x)=\rho(\varphi(x))=a \varphi(x)+b .
$$

Since $\varphi$ and $\tilde{\varphi}$ are increasing, $a$ must be positive.

We could select a unique $\varphi$ representing a family of functions by stipulating, for example, that $\varphi(1 / 2)=0$ and $\varphi(3 / 4)=1$. This still leaves uncountably many strictly increasing bijections of ]0,1[ onto $\mathbb{R}$ which are not affine functions of each other; so there are uncountably many families of functions satisfying the requirements $1^{\prime}, 2-4,5^{\prime}, 6,7$, $8^{\prime}, 10$, and 11 .

Note that addition of a constant $b$ to $\varphi(x)$ does not change the functions defined by (5) and (6) at all, because the constant simply cancels out. The multiplication by $a$ corresponds to a reparametrization of the family $\left\{f^{t}\right\}$ given by (5). It again has no effect on (6), however.

We note that we may also allow $\varphi$ to be decreasing. The theorem would still hold, with the obvious modifications of (8). Everything else could remain unchanged.

5. A further property: symmetry with respect to $y=1-x$; the second characterization theorem; Y.-K. Ng's example. In [12] a further "reasonable property" is an invariance with respect to replacing all probabilities $x$ by the complementary probabilities $1-x$ :

Property 9 (Complementarity). $F\left(1-x_{1}, 1-y_{1}, 1-x_{2}\right)=1-F\left(x_{1}, y_{1}, x_{2}\right)$.

This equation is rather intuitive. Consider, for instance, the connection between changes in employment and unemployment rates. If the change from $x_{1}$ to $x_{2}$ is equivalent to the change from $y_{1}$ to $y_{2}$ then the change from $1-x_{1}$ to $1-x_{2}$ should also be equivalent to the change from $1-y_{1}$ to $1-y_{2}$. A geometric interpretation of Property 9 in conjunction with Property 10 can be derived as follows. Property 9 states that $F\left(x_{1}, y_{1}, x_{2}\right)=y_{2}$ implies $F\left(1-x_{1}, 1-y_{1}, 1-x_{2}\right)=1-y_{2}$, and vice versa. By Property 10, the last equation is equivalent to $F\left(1-y_{1}, 1-x_{1}, 1-y_{2}\right)=1-x_{2}$. Thus we have as an equivalent formulation:

$$
F\left(x_{1}, y_{1}, x_{2}\right)=y_{2} \quad \text { implies } \quad F\left(1-y_{1}, 1-x_{1}, 1-y_{2}\right)=1-x_{2}
$$




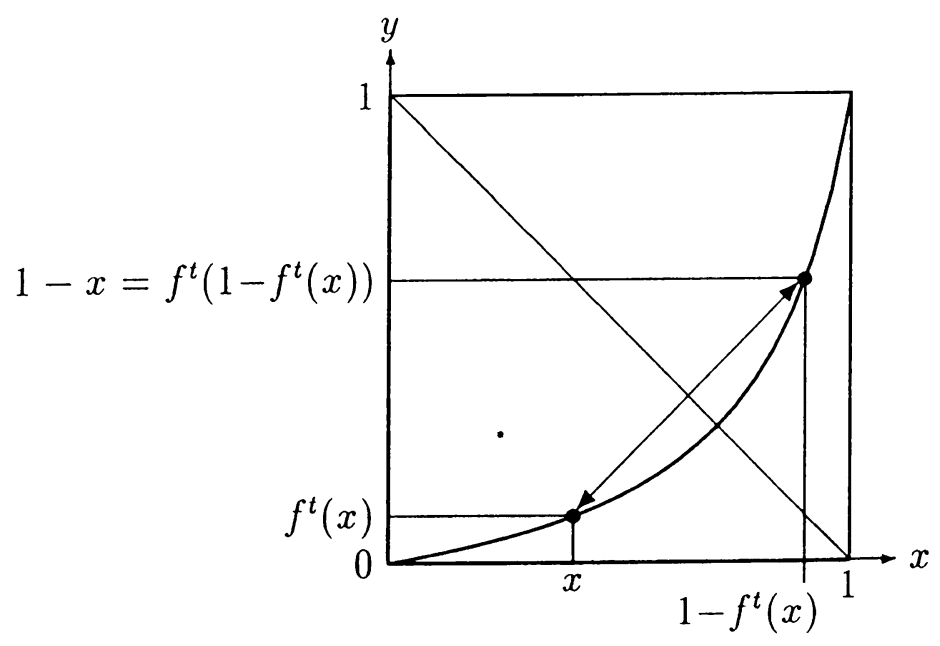

FIG. 2. Reflection with respect to the line $x+y=1$

Now consider an arbitrary function $f^{t}$ and let $\left(x_{1}, y_{1}\right)$ be the point of intersection of its graph with the line $x+y=1$. (This point exists because of Properties 6 and $8^{\prime}$.) We have then, for all $x$ and $y$,

$$
f^{t}(x)=F\left(x_{1}, y_{1}, x\right)=y
$$

implies

$$
1-x=F\left(1-y_{1}, 1-x_{1}, 1-y\right)=F\left(x_{1}, y_{1}, 1-y\right)=f^{t}(1-y) .
$$

The next-to-last equation follows from $x_{1}+y_{1}=1$. Thus, we get the following relation from Properties 9 and 10:

$$
f^{t}(x)=y \quad \text { implies } \quad f^{t}(1-y)=1-x
$$

or

$$
f^{t}\left(1-f^{t}(x)\right)=1-x .
$$

Geometrically, with every point $(x, y)=\left(x, f^{t}(x)\right)$, also its mirror image with respect to the $135^{\circ}$ line, $(1-y, 1-x)=\left(1-f^{t}(x), 1-x\right)$, lies on the graph of $f^{t}$ (see Fig. 2), that is, the graph of each $f^{t}$ is symmetric with respect to the line $y=1-x$.

To check the converse implication from (12) and from Property 10 to Property 9, remember that $F\left(x_{1}, y_{1}, x_{2}\right)=y_{2}$ means that there is an $f^{t}$ in the family whose graph contains both points $\left(x_{1}, y_{1}\right)$ and $\left(x_{2}, y_{2}\right)$. By the symmetry of $f^{t}$, or by (11), the same $f^{t}$ contains the points $\left(1-y_{1}, 1-x_{1}\right)$ and $\left(1-y_{2}, 1-x_{2}\right)$, that is, we conclude that $F\left(1-y_{1}, 1-x_{1}, 1-y_{2}\right)=1-x_{2}$. So we have $(10)$, which, as we have seen above, is equivalent to Property 9 if Property 10 is also supposed.

We will now determine the general function $f^{t}$, of the form (5), which satisfies (12). First notice that, for each fixed $t, f^{t}$ is a bijection of ]0,1[ onto ]0,1[. (We are here ignoring the values $f^{t}(0)=0$ and $f^{t}(1)=1$.) We can write $(12)$ with $y=f^{t}(x)$, that is, $x=\left(f^{t}\right)^{-1}(y)=f^{-t}(y)$, as

$$
f^{t}(1-y)=1-f^{-t}(y) .
$$


In view of (5), this is equivalent to

$$
\varphi^{-1}(\varphi(1-y)+t)=1-\varphi^{-1}(\varphi(y)-t) .
$$

For $t=\varphi(y)$ this reduces to

$$
\varphi^{-1}(\varphi(1-y)+\varphi(y))=1-\varphi^{-1}(\varphi(y)-\varphi(y))=1-\varphi^{-1}(0) .
$$

Thus we have, for all $y \in] 0,1[$,

$$
\varphi(1-y)+\varphi(y)=\varphi\left(1-\varphi^{-1}(0)\right)
$$

which is constant. By setting $y=1 / 2$ we see that the constant is $2 \varphi(1 / 2)$. Since we may add an arbitrary constant to $\varphi$ without changing the functions $f^{t}$ (cf. the discussion after Theorem 1), we can without loss of generality make the following simplifying assumption:

$$
\varphi(1 / 2)=0
$$

Our condition now becomes

$$
\varphi(1-y)+\varphi(y)=0 \quad \text { for all } y \in] 0,1[
$$

With $z=\frac{1}{2}-y$ this can be written as

$$
\varphi\left(\frac{1}{2}+z\right)=-\varphi\left(\frac{1}{2}-z\right)
$$

This means that the graph of $\varphi$ is symmetric about the point $\left(\frac{1}{2}, 0\right)=\left(\frac{1}{2}, \varphi\left(\frac{1}{2}\right)\right)$. Thus the bijection $g:]-\frac{1}{2}, \frac{1}{2}[\rightarrow \mathbb{R}$ defined by

$$
g(z)=\varphi\left(\frac{1}{2}+z\right)
$$

is odd:

$$
g(-z)=-g(z)
$$

Conversely, if we start with an arbitrary continuous strictly increasing odd bijection $g:]-\frac{1}{2}, \frac{1}{2}[\rightarrow \mathbb{R}$, the function

$$
\varphi(y):=g\left(y-\frac{1}{2}\right)
$$

will always satisfy (13). Indeed, from (16) we get

$$
\varphi^{-1}(s)=\frac{1}{2}+g^{-1}(s) \text {. }
$$

We have

$$
\varphi(1-y)=g\left(\frac{1}{2}-y\right)=-g\left(y-\frac{1}{2}\right),
$$

because $g$ is odd, and therefore

$$
\varphi^{-1}(\varphi(1-y)+t)=\frac{1}{2}+g^{-1}(\varphi(1-y)+t)=\frac{1}{2}+g^{-1}\left(-g\left(y-\frac{1}{2}\right)+t\right),
$$


while

$$
1-\varphi^{-1}(\varphi(y)-t)=\frac{1}{2}-g^{-1}(\varphi(y)-t)=\frac{1}{2}-g^{-1}\left(g\left(y-\frac{1}{2}\right)-t\right) .
$$

The right-hand sides of the last two chains of equations are equal, since with $g$ also $g^{-1}$ is odd. Thus (13) is satisfied and so is (12) for all $x \in] 0,1[$ and $t \in \mathbb{R}$.

We have also, for all $t \in \mathbb{R}$,

$$
\begin{aligned}
& \left.f^{t}(x)=\varphi^{-1}(t+\varphi(x))=\frac{1}{2}+g^{-1}\left(t+g\left(x-\frac{1}{2}\right)\right) \quad \text { for } x \in\right] 0,1[, \\
& f^{t}(0)=0, \quad f^{t}(1)=1 .
\end{aligned}
$$

The boundary equations for 0 and 1 come from Property 6 and make (12) valid also for $x=0$ and $x=1$ :

$$
f^{t}\left(1-f^{t}(0)\right)=f^{t}(1)=1-0, \quad f^{t}\left(1-f^{t}(1)\right)=f^{t}(0)=1-1 .
$$

Substitution of (16) and (17) into (6) gives the representation

$$
\begin{gathered}
y_{2}=F\left(x_{1}, y_{1}, x_{2}\right)=\frac{1}{2}+g^{-1}\left(g\left(y_{1}-\frac{1}{2}\right)-g\left(x_{1}-\frac{1}{2}\right)+g\left(x_{2}-\frac{1}{2}\right)\right) \quad \text { for } 0<x_{2}<1, \\
F\left(x_{1}, y_{1}, 0\right)=0, \quad F\left(x_{1}, y_{1}, 1\right)=1 .
\end{gathered}
$$

So we have proved the following theorem.

Theorem 2. The general families of functions satisfying the requirements $1^{\prime}, 2-4,5^{\prime}, 6$, $7,8^{\prime}$, and $9-11$ are given by (18) and (19), where $g$ is an arbitrary continuous, strictly increasing, and odd bijective mapping from $]-\frac{1}{2}, \frac{1}{2}[$ onto $\mathbb{R}$.

Like the function $\varphi$ in Theorem 1 , the function $g$ in Theorem 2 can be subjected to an arbitrary affine transformation $g(z) \mapsto a \cdot g(z)+b$ with $a \neq 0$ without affecting the family $f^{t}$. However, for $b \neq 0$ these transformations do not preserve oddness. In fact, the requirement that $g$ be odd can be weakened to the symmetry of $g$ about the point $(0, g(0)): g(-z)-g(0)=-(g(z)-g(0))$. (Similarly, it does not matter whether $g$ is increasing or decreasing.) The more restrictive formulation in Theorem 2 comes from the simplifying assumption (14) that $\varphi(1 / 2)=g(0)=0$.

$\mathrm{Ng}[12]$ presents an example that meets all requirements 1 (really $1^{\prime}$ ) and $2-11$. We show how the solution in [12] fits into the framework of the above theorem. That solution is

$$
f^{[i]}(x)=\frac{(i+1) x}{2 x+i-1}
$$

or, in terms of $(1)$,

$$
y_{2}=F\left(x_{1}, y_{1}, x_{2}\right)=\frac{x_{2} y_{1}\left(1-x_{1}\right)}{x_{1}\left(1-x_{2}\right)+y_{1}\left(x_{2}-x_{1}\right)}
$$

see Fig. 3 on p. 488. To see that these functions are of the forms (5) and (6), respectively, choose $\varphi(x)=\ln (x /(1-x))$, that is, $\varphi^{-1}(s)=1 /\left(1+e^{-s}\right)$, and the reparametrization

$$
t=\mu(i)=\ln \frac{i+1}{i-1} \text {. }
$$




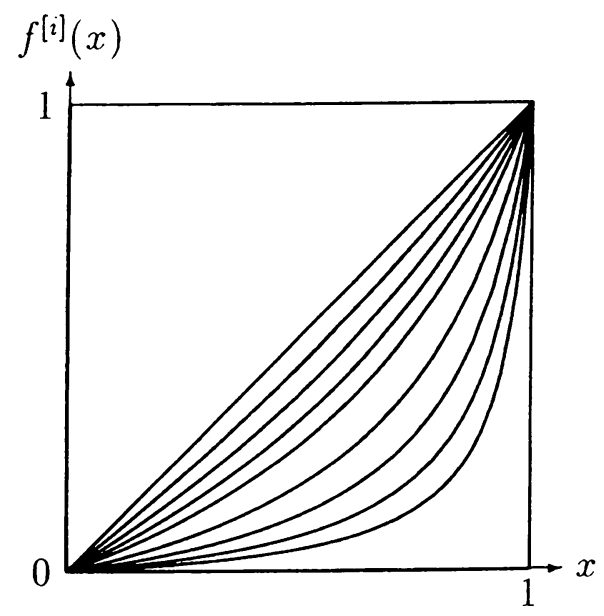

FiG. 3. The functions $f^{t}$ in Ng's example, defined by (21), for $t=0$, $-0.25,-0.5,-0.75,-1,-1.5,-2,-2.5$, and -3 .

Then indeed

$$
f^{t}(x)=\varphi^{-1}(t+\varphi(x))=\frac{1}{1+e^{-t-\ln (x /(1-x))}}=\frac{1}{1+e^{-t} \cdot \frac{1-x}{x}}=\frac{x}{x+e^{-t}(1-x)}
$$

and

$$
f^{[i]}(x)=f^{\mu(i)}(x)=\frac{x}{x+\frac{i-1}{i+1}(1-x)}=\frac{(i+1) x}{(i+1) x+(i-1)(1-x)}=\frac{(i+1) x}{2 x+i-1},
$$

as it should be. Also

$$
\begin{aligned}
F\left(x_{1}, y_{1}, x_{2}\right) & =\varphi^{-1}\left(\varphi\left(y_{1}\right)-\varphi\left(x_{1}\right)+\varphi\left(x_{2}\right)\right)=\frac{1}{1+\exp \left(-\ln \frac{y_{1}}{1-y_{1}}+\ln \frac{x_{1}}{1-x_{1}}-\ln \frac{x_{2}}{1-x_{2}}\right)} \\
& =1 /\left(1+\frac{1-y_{1}}{y_{1}} \frac{x_{1}}{1-x_{1}} \frac{1-x_{2}}{x_{2}}\right)=\frac{x_{2} y_{1}\left(1-x_{1}\right)}{x_{2} y_{1}\left(1-x_{1}\right)+\left(1-y_{1}\right) x_{1}\left(1-x_{2}\right)} \\
& =\frac{x_{2} y_{1}\left(1-x_{1}\right)}{x_{1}\left(1-x_{2}\right)+y_{1}\left(x_{2}-x_{1}\right)},
\end{aligned}
$$

again as required. Let us finally check that $f^{t}(x)$ is indeed of the form (18). We define the function $g$ by

$$
g(z)=\ln \frac{1+2 z}{1-2 z} \quad \text { for }-1 / 2<z<1 / 2 .
$$

This function is odd: $g(-z)=\ln ((1-2 z) /(1+2 z))=-g(z)$, and its inverse is $g^{-1}(s)=$ $\frac{1}{2}\left(e^{s}-1\right) /\left(e^{s}+1\right)=\frac{1}{2} \tanh \frac{s}{2}$. We have $\frac{1}{2}+g^{-1}(s)=\frac{1}{2}\left(1+\tanh \frac{s}{2}\right)=1 /\left(e^{-s}+1\right)$, and thus

$$
\begin{aligned}
\frac{1}{2}+g^{-1}\left(t+g\left(x-\frac{1}{2}\right)\right) & =1 /\left(\exp \left(-t-g\left(x-\frac{1}{2}\right)\right)+1\right) \\
& =1 /\left(\exp \left(-t-\ln \frac{x}{1-x}\right)+1\right) \\
& =\frac{1}{e^{-t} \cdot \frac{1-x}{x}+1}=\frac{x}{(1-x) e^{-t}+x}=f^{t}(x) .
\end{aligned}
$$


As we see, here also $f^{t}(0)=0$ and $f^{t}(1)=1$ are contained in the same formula.

Although the function given by $g(z)=\ln ((1+2 z) /(1-2 z))$ is simple, there is nothing special about it that would imply that the functions $f^{t}$ are in some sense natural or best solutions, as was conjectured by $\mathrm{Ng}[12]$. However, if we substitute $\varphi(x)=\ln (x /(1-x))$ into (7) and take exponentials on both sides of the equation we get the following condition for equivalent changes in probabilities:

$$
\frac{y_{1}}{1-y_{1}}: \frac{y_{2}}{1-y_{2}}=\frac{x_{1}}{1-x_{1}}: \frac{x_{2}}{1-x_{2}}
$$

and this could be explained by saying that the "odds" $x /(1-x)$ have to change proportionally.

\section{A last property: convexity; further characterization theorems and ex-}

amples. For the case that Properties 1-11 might not characterize equivalent changes uniquely, the author suggests in [12] that "we may wish to adopt further reasonable properties so as to narrow down the permissible functions defining equivalent changes, preferably to a unique function $F$ for a unique family $f^{[i]}$." He proposes the Property 12 that "for any $f^{[i]}$ whose graph lies below/above the $y=x$ line, $d y / d x=\left(f^{[i]}\right)^{\prime}$ be (strictly) increasing/decreasing throughout" (again slightly rephrased). It is clear that, for differentiable functions, this is the (strict) convexity/concavity of $f^{[i]}$, and that is what we will call it. Without supposing differentiability (we will prove, though, that the $f^{i}$ are continuously differentiable) we will show that this requirement does not narrow down the permissible functions to a unique family of functions either, and we will determine all functions satisfying the requirements $1-12$. We state two variants of Property 12 :

Property 12' (Convexity). Each $f^{t}$ is convex on ]0, $1[$ for $t<0$.

Property 12" (Strict Convexity). Each $f^{t}$ is strictly convex on $] 0,1[$ for $t<0$.

By Property 10, $f^{t}$ will then be (strictly) concave for $t>0$. Of course, $f^{0}$ is the linear function $y=x$.

Before we investigate how to modify Theorem 1 to accommodate this additional property we will show that it implies that $f^{t}, \varphi$, and $\varphi^{-1}$ are continuously differentiable. Recall that we have already obtained from the other properties the representation

$$
f^{t}(x)=\varphi^{-1}(\varphi(x)+t)
$$

where $\varphi$ was a strictly increasing continuous function, with the extension (8). In this section we will more often deal with the inverse function $\varphi^{-1}$, and thus it will be conveinient to write (5) as

$$
f^{t}(x)=\Phi\left(\Phi^{-1}(x)+t\right)
$$

where $\Phi=\varphi^{-1}$ is a strictly increasing and continuous bijection $\Phi: \mathbb{R} \cup\{-\infty, \infty\} \rightarrow[0,1]$.

Now we have in addition that $f^{t}$ is concave on $] 0,1[$ if $t>0$. Therefore, the right derivative $\left(f^{t}\right)_{+}^{\prime}$ of $f^{t}$ exists and is finite for all $\left.x \in\right] 0,1[$ :

$$
\left(f^{t}\right)_{+}^{\prime}(x)=\lim _{h \rightarrow 0^{+}} \frac{f^{t}(x+h)-f^{t}(x)}{h}<\infty .
$$


For arbitrary $x$ and $h$ with $0<x<x+h<1$ there exist $s$ and $\delta>0$ such that $x=\Phi(s)$, $x+h=\Phi(s+\delta)$. So we can write

$$
\left(f^{t}\right)_{+}^{\prime}(x)=\lim _{\delta \rightarrow 0^{+}} \frac{\Phi(s+t+\delta)-\Phi(s+t)}{\Phi(s+\delta)-\Phi(s)}=\lim _{\delta \rightarrow 0^{+}} \frac{(\Phi(s+t+\delta)-\Phi(s+t)) / \delta}{(\Phi(s+\delta)-\Phi(s)) / \delta} .
$$

Furthermore, since $\Phi$ is monotonic, it is differentiable at some point $s_{0}$ (even almost everywhere). So for $s=s_{0}$ the limit of the denominator

$$
\lim _{\delta \rightarrow 0^{+}} \frac{\Phi\left(s_{0}+\delta\right)-\Phi\left(s_{0}\right)}{\delta}
$$

exists. By (22), also the limit of the numerator

$$
\lim _{\delta \rightarrow 0^{+}} \frac{\Phi\left(s_{0}+t+\delta\right)-\Phi\left(s_{0}+t\right)}{\delta}
$$

exists. Since $t$ was arbitrary, $\Phi$ is thus differentiable from the right everywhere. So the limits of both the numerator and the denominator in (22) exist for all $s$ and $t$. Moreover, the limit of the denominator could be 0 at $s=s_{0}$ only if the limit of the numerator would be 0 too, that is, if $\Phi_{+}^{\prime}\left(s_{0}+t\right)=0$ for all $t$. So $\Phi$ would have to be constant, but it is strictly increasing; therefore, $\Phi_{+}^{\prime}(z) \neq 0$ for all $z \in \mathbb{R}$.

We differentiate $f^{t}$ from the right with respect to $t$, using the representation $\left(5^{\prime}\right)$, and since $\Phi_{+}^{\prime}$ exists everywhere, so does

$$
\Phi_{+}^{\prime}\left(\Phi^{-1}(x)\right)=\left.\frac{\partial f^{t}(x)}{\partial t}\right|_{t \rightarrow 0^{+}}=\lim _{t \rightarrow 0^{+}} \frac{f^{t}(x)-f^{0}(x)}{t}=\lim _{t \rightarrow 0^{+}} \frac{f^{t}(x)-x}{t} .
$$

We denote this function of $x$ by $\sigma$. As the limit of the concave functions $\left(f^{t}(x)-x\right) / t$, the function $\sigma$ is clearly concave, and thus continuous (cf. $[10,14]$ ). Therefore, the function $\sigma(\Phi(s))=\Phi_{+}^{\prime}(s)$ is continuous on $\mathbb{R}$. It follows that $\Phi$ is differentiable, $\Phi_{+}^{\prime}=\Phi^{\prime}>0$, and $\Phi^{\prime}$ is continuous on $\mathbb{R}$.

Since $\Phi^{\prime}$ is continuous and nowhere 0 , also $\Phi^{-1}$ is continuously differentiable. Therefore, we have proved the following theorem.

THEOREM 3. If a family of functions satisfies the requirements $1^{\prime}, 2-4,5^{\prime}, 6,7,8^{\prime}, 10,11$, and $12^{\prime}$ or $12^{\prime \prime}$, then the functions $\varphi$ and $\Phi=\varphi^{-1}$ in (5) are continuously differentiable, and thus the functions $f^{t}$ are themselves continuously differentiable.

Given these differentiability properties, it is not difficult to characterize the functions $\varphi$ that give rise to convex and concave functions $f^{t}$. From (22),

$$
\left(f^{t}\right)^{\prime}(x)=\frac{\Phi^{\prime}(s+t)}{\Phi^{\prime}(s)}
$$

where $x=\Phi(s)$. The derivatives of (strictly) concave functions are (strictly) decreasing. So, by Property $12^{\prime}$ or $12^{\prime \prime}, d y / d x=\left(f^{t}\right)^{\prime}(x)$ has to be (strictly) decreasing in $x$ for fixed $t>0$ and, since $\varphi$ in $s=\varphi(x)$ is strictly increasing,

$$
\frac{\Phi^{\prime}(s+t)}{\Phi^{\prime}(s)}
$$




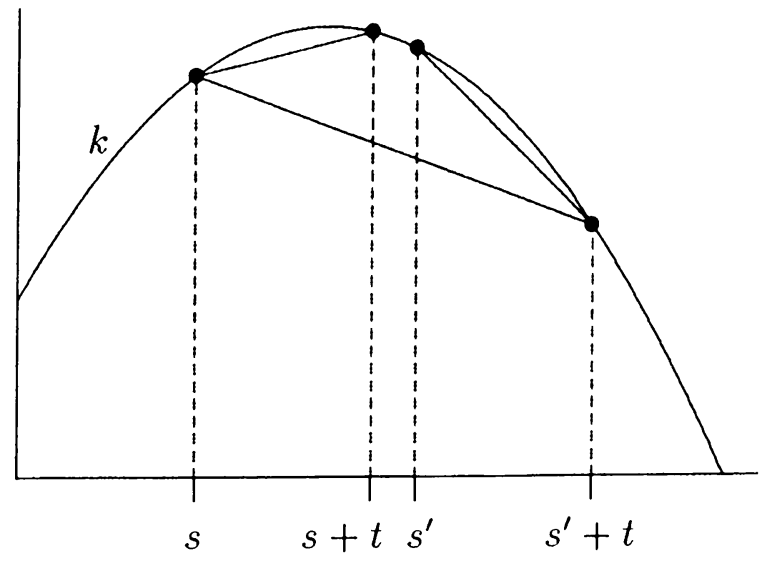

FIG. 4. Concavity of $k$

will be (strictly) decreasing in $s$ for all fixed $t>0$. We take logarithms, remembering that $\Phi^{\prime}$ is positive, and introduce the continuous function $k: \mathbb{R} \rightarrow \mathbb{R}$ defined by

$$
k(s)=\ln \left(\Phi^{\prime}(s)\right) .
$$

Thus,

$$
\ln \left(\Phi^{\prime}(s+t)\right)-\ln \left(\Phi^{\prime}(s)\right)=k(s+t)-k(s)
$$

is (strictly) decreasing in $s$, for $t>0$. But (cf. [10] and Fig. 4), $k(s+t)-k(s)$ decreases (strictly) in $s$ for all fixed positive $t$ exactly if $k$ is (strictly) concave. Indeed, for (strictly) concave functions, the slope of the chord from one point of the graph to a variable second point (strictly) decreases. So, for $t>0$ and $s^{\prime}>s$

$$
\frac{k(s+t)-k(s)}{t} \geq \frac{k\left(s^{\prime}+t\right)-k(s)}{s^{\prime}+t-s} \geq \frac{k\left(s^{\prime}+t\right)-k\left(s^{\prime}\right)}{t}
$$

with strict inequalities in the case of Property $12^{\prime \prime}$. On the other hand, by setting $s^{\prime}=s+t$ in $k(s+t)-k(s) \geq k\left(s^{\prime}+t\right)-k\left(s^{\prime}\right)$ we get the Jensen inequality $2 k(s+t) \geq k(s)+k(s+2 t)$, which, for continuous functions $k$, implies that $k$ is concave (strictly concave in the case of strict inequality). Now, from $(23), \Phi^{\prime}(s)=e^{k(s)}$ and by $(8), \Phi(-\infty)=\varphi^{-1}(-\infty)=0$. So we get

$$
\varphi^{-1}(s)=\Phi(s)=\int_{-\infty}^{s} e^{k(z)} d z
$$

and

$$
\varphi^{-1}(\infty)=\Phi(\infty)=\int_{-\infty}^{\infty} e^{k(z)} d z=1
$$

provided that the improper integrals exist. (This is only a question about the limit as the bounds go to $\infty$, since, like all concave functions, $k$ is continuous (see [10]), and therefore $e^{k(z)}$ is integrable.) Existence of the improper integrals is ensured as long as $k(z)$ is not (weakly) monotonic throughout, that is, in addition to being concave it is first strictly 


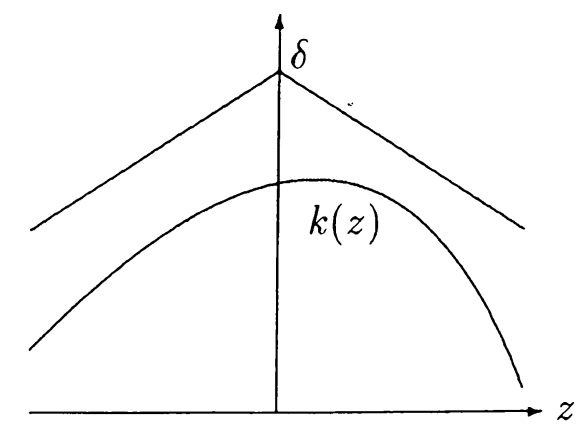

FIG. 5. A concave function majorized by a "roof" function $\delta-\gamma|z|$

increasing, then possibly constant for some interval, and finally strictly decreasing (it is unimodal; the constant part may be missing). Then, by concavity, $k(z)$ is majorized by some "roof" function of the form $-\gamma|z|+\delta$ with $\gamma>0$ (see Fig. 5), that is,

$$
0<e^{k(z)} \leq e^{-\gamma|z|+\delta}
$$

and

$$
\int_{-\infty}^{s} e^{k(z)} d z \leq \int_{-\infty}^{\infty} e^{k(z)} d z \leq \int_{-\infty}^{\infty} e^{-\gamma|z|+\delta} d z=2 e^{\delta} / \gamma<\infty .
$$

On the other hand, if $k(z)$ is, say, monotonically increasing, then there are constants $k_{0}$ and $z_{0}$ such that $k(z) \geq k_{0}$ for all $z \geq z_{0}$, and the integral

$$
\int_{-\infty}^{\infty} e^{k(z)} d z \geq \int_{z_{0}}^{\infty} e^{k_{0}} d z
$$

is clearly infinite.

Condition (25) is just a normalizing condition. Thus we may start with an arbitrary concave or strictly concave function $h(z)$ for which the improper integral

$$
C:=\int_{-\infty}^{\infty} e^{h(z)} d z
$$

exists. Then the function $k(z):=h(z)-\ln C$ is also (strictly) concave. We get

$$
\varphi^{-1}(s)=\Phi(s)=\int_{-\infty}^{s} e^{h(z)-\ln C} d z=\frac{1}{C} \cdot \int_{-\infty}^{s} e^{h(z)} d z
$$

and (25) will be fulfilled. Since the function $\Phi: \mathbb{R} \rightarrow] 0,1[$ defined in this way is continuous, differentiable and strictly increasing, and since it fulfills

$$
\lim _{s \rightarrow-\infty} \Phi(s)=0 \text { and } \lim _{s \rightarrow \infty} \Phi(s)=1
$$

by the definition of improper integrals, it is a bijection onto ]0,1[. It follows that its inverse function $\varphi=\Phi^{-1}$ is also a continuous and strictly increasing bijection and fulfills 
(8), thus satisfying all conditions of Theorem 1 . Furthermore, $\Phi^{\prime}(s+t) / \Phi^{\prime}(s)$ decreases (strictly) for every fixed $t>0$. (For this the condition that $k$, or $h=k+\ln C$, be (strictly) concave was necessary and sufficient.) So, for $t>0$, every $f^{t}$ as given by (5) will be (strictly) concave, and Property $12^{\prime}$ or $12^{\prime \prime}$ will also be fulfilled. Thus we have proved the following theorem.

THEOREM 4. The general family of functions for which the requirements $1^{\prime}, 2-4,5^{\prime}, 6$, $7,8^{\prime}, 10,11$, and $12^{\prime}$ (or $12^{\prime \prime}$ ) are satisfied is given by the representation (5), with

$$
\begin{gathered}
\varphi^{-1}(s)=\frac{1}{C} \int_{-\infty}^{s} e^{h(z)} d z \quad \text { for } s \in \mathbb{R} \\
\varphi^{-1}(-\infty)=0, \quad \varphi^{-1}(+\infty)=1
\end{gathered}
$$

Here, $C=\int_{-\infty}^{\infty} e^{h(z)} d z>0$, and $h: \mathbb{R} \rightarrow \mathbb{R}$ is an arbitrary concave (resp. strictly concave) function that is strictly increasing at a point and strictly decreasing at another point. (This ensures the existence of both improper integrals.)

We may of course consider the convexity property in conjunction with the symmetry property 9 of the previous section. The additional condition that we have to satisfy is that the function $g(z)=\varphi\left(\frac{1}{2}+z\right)$ defined by (15) be odd. This is tantamount to requiring that the inverse function,

$$
g^{-1}(s)=\varphi^{-1}(s)-\frac{1}{2}=\Phi(s)-\frac{1}{2}
$$

be odd. Differentiating this by $s$, we get

$$
\Phi^{\prime}(s)=\left(g^{-1}\right)^{\prime}(s)
$$

If $g^{-1}$ is odd then $\left(g^{-1}\right)^{\prime}$ is even and so is $\Phi^{\prime}$. Thus, by (23), also $k$ is even. The function $h$ differs from $k$ only by a constant and is therefore also even.

All the above arguments are reversible. Indeed, it is easy to check that for an even function $h,(26)$ in Theorem 4 will give rise to a function $\varphi^{-1}$ that makes $g^{-1}(s)=$ $\varphi^{-1}(s)-1 / 2$ odd, because $\varphi^{-1}(-s)-1 / 2=-\left(\varphi^{-1}(s)-1 / 2\right)$ is equivalent to

$$
\varphi^{-1}(-s)+\varphi^{-1}(s)=1
$$

and this follows from

$$
\begin{aligned}
\varphi^{-1}(-s)+\varphi^{-1}(s) & =\frac{1}{C} \int_{-\infty}^{-s} e^{h(z)} d z+\frac{1}{C} \int_{-\infty}^{s} e^{h(z)} d z \\
& =-\frac{1}{C} \int_{\infty}^{s} e^{h(-\hat{z})} d \hat{z}+\frac{1}{C} \int_{-\infty}^{s} e^{h(z)} d z \\
& =\frac{1}{C} \int_{s}^{\infty} e^{h(\hat{z})} d \hat{z}+\frac{1}{C} \int_{-\infty}^{s} e^{h(z)} d z=\frac{1}{C} \int_{-\infty}^{\infty} e^{h(z)} d z=1
\end{aligned}
$$

Therefore, the family $\left\{f^{t}\right\}$ will also fulfill Property 9 by Theorem 2 . So we have proved the following theorem. 
THEOREM 5. The general family of functions for which the requirements $1^{\prime}, 2-4,5^{\prime}, 6$, $7,8^{\prime}, 9-11$, and $12^{\prime}$ (or $12^{\prime \prime}$ ) are satisfied is given by the representation (5), with

$$
\begin{gathered}
\varphi^{-1}(s)=\frac{1}{C} \int_{-\infty}^{s} e^{h(z)} d z, \quad \text { for } s \in \mathbb{R}, \\
\varphi^{-1}(-\infty)=0, \quad \varphi^{-1}(+\infty)=1 .
\end{gathered}
$$

Here $C=\int_{-\infty}^{\infty} e^{h(z)} d z>0$, and $h: \mathbb{R} \rightarrow \mathbb{R}$ is an arbitrary nonconstant even concave (resp. strictly concave) function. (The existence of both improper integrals is guaranteed.)

Note that, in the case of even concave functions, it is enough to exclude the constant functions in order to ensure that the improper integrals exist.

Let us discuss the uniqueness of the functions $h$ in Theorems 4 and 5 for a given family $\left\{f^{t}\right\}$. Recall from the remarks after Theorem 1 that the function $\varphi$ is unique up to affine transformations $\varphi(x) \mapsto a \cdot \varphi(x)+b$. In terms of $\varphi^{-1}(s)=\Phi(s)$, this means that affine transformations of the variable $s$ in the domain of $\Phi$, and only these, leave the family $\left\{f^{t}\right\}$ unchanged. We may thus go from $\Phi(s)$ to $\tilde{\Phi}(s)=\Phi(a s+b)$, and by (23) and (26), from $h(s)$ to $\tilde{h}(s)=h(a s+b)$, for $a \neq 0$. Addition of a constant to $h$ also has no effect, since the division by the normalizing factor $C$ cancels it. The class of all functions $h$ that yield the same family $\left\{f^{t}\right\}$ by Theorems 4 and 5 is therefore

$$
\{\tilde{h}(s)=h(a s+b)+c \mid a \neq 0 ; b, c \in \mathbb{R}\},
$$

for some fixed function $h$. Note that for $b \neq 0$ these transformations do not preserve evenness. Thus the function $h$ in Theorem 5 actually need not be even, as long as it is symmetric about some point $\gamma: h(\gamma+s)=h(\gamma-s)$. The more restrictive formulation in Theorem 5 comes from the simplifying assumption (14) in Sec. 5 that $\varphi(1 / 2)=0$, i.e., $\Phi(0)=1 / 2$; cf. the discussion after Theorem 2 .

Again there are uncountably many strictly concave even functions $h$ that differ in more than a linear transformation of the variable or an addition of a constant (see Example 3 below for uncountably many concave ones), and so there are still uncountably many families of functions satisfying all our requirements. We conclude with a few further examples of such families.

1. If $h(z)=-z^{2} / 2$, which is even and strictly concave, then

$$
C=\int_{-\infty}^{\infty} e^{-z^{2} / 2} d z=\sqrt{2 \pi}
$$

and

$$
\varphi^{-1}(s)=\frac{1}{\sqrt{2 \pi}} \int_{-\infty}^{s} e^{-z^{2} / 2} d z
$$

as in the normal (Gaussian) probability distribution. The corresponding family of functions is shown in Fig. 6a.

2. The following function is concave, but not strictly concave, and not even. It will thus give rise to a family of curves that is not symmetric about the line $x+y=1$ :

$$
h(z)= \begin{cases}2 z & \text { for } z \leq 0 \\ -z & \text { for } z \geq 0\end{cases}
$$


$f^{t}(x)$

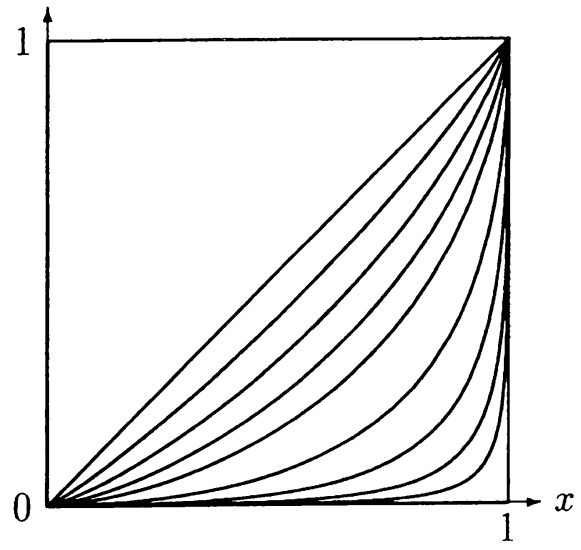

(a)

$f^{t}(x)$

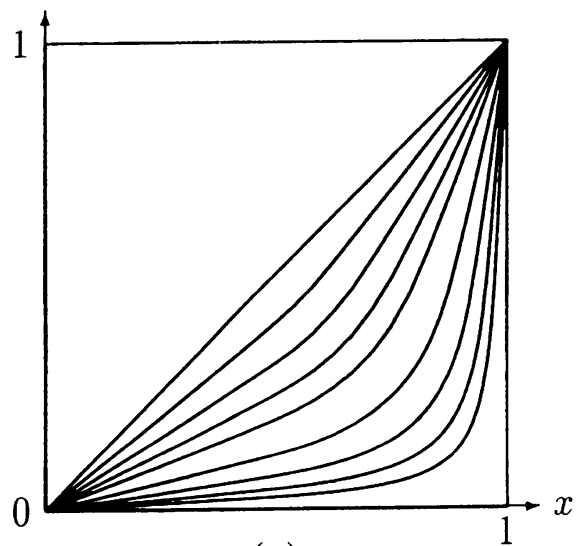

(c)

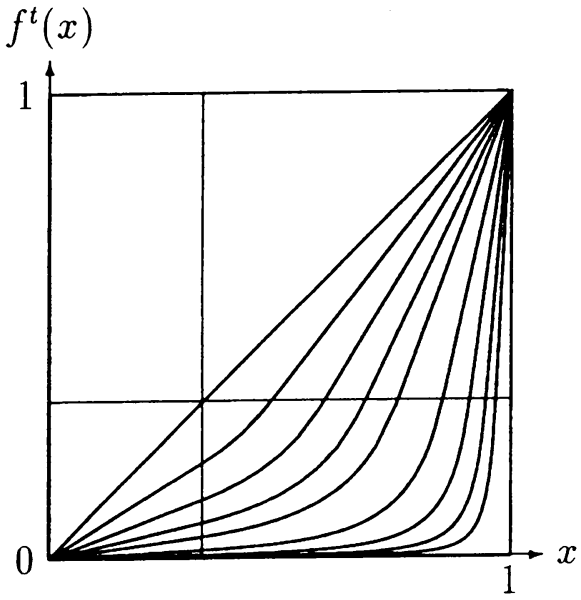

(b)

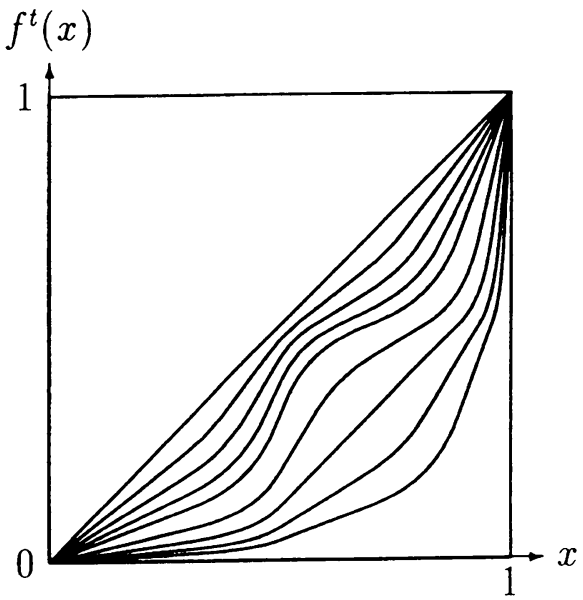

(d)

FIG. 6. Examples of families of functions $f^{t}$ defining equivalent changes of probabilities. Each figure shows the functions $f^{t}$ for $t=0$, $-0.25,-0.5,-0.75,-1,-1.5,-2,-2.5$, and -3 . The functions $f^{t}$ for positive $t$ are obtained by reflecting $f^{-t}$ on the diagonal $y=$ $x$. (a) The family corresponding to the normal distribution; (b) a nonsymmetric family resulting from (27); (c) and (d): the families resulting from $h(z)$ as given by (28), for $\alpha=0.9$ and $\alpha=-1$, respectively.

We obtain

$$
\begin{gathered}
C=\int_{-\infty}^{\infty} e^{h(z)} d z=\int_{-\infty}^{0} e^{2 z} d z+\int_{0}^{\infty} e^{-z} d z=e^{0} / 2-0-0+e^{0}=3 / 2, \\
\varphi^{-1}(s)=\Phi(s)= \begin{cases}\frac{1}{3} e^{2 s} & \text { for } s \leq 0 \\
1-\frac{2}{3} e^{-s} & \text { for } s>0 .\end{cases}
\end{gathered}
$$


The two segments join smoothly because

$$
\begin{aligned}
& \lim _{s \rightarrow 0^{+}} \Phi(s)=\lim _{s \rightarrow 0^{-}} \Phi(s)=\frac{1}{3}=\Phi(0), \\
& \lim _{s \rightarrow 0^{+}} \Phi^{\prime}(s)=\lim _{s \rightarrow 0^{-}} \Phi^{\prime}(s)=\frac{2}{3}=\Phi^{\prime}(0) .
\end{aligned}
$$

Here we give also $f^{t}(x)$ as calculated from (5):

$$
f^{t}(x)= \begin{cases}e^{2 t} x & \text { for } 0 \leq x \leq \frac{1}{3} \\ \frac{4}{27} e^{2 t} /(1-x)^{2} & \text { for } \frac{1}{3} \leq x \leq 1-\frac{2}{3} e^{t}, \quad \text { for } t \leq 0 \\ 1-e^{-t}(1-x) & \text { for } 1-\frac{2}{3} e^{t} \leq x \leq 1\end{cases}
$$

and

$$
f^{t}(x)= \begin{cases}e^{2 t} x & \text { for } 0 \leq x \leq \frac{1}{3} e^{-2 t} \\ 1-2 e^{-t} / \sqrt{27 x} & \text { for } \frac{1}{3} e^{-2 t} \leq x \leq \frac{1}{3}, \quad \text { for } t>0 \\ 1-e^{-t}(1-x) & \text { for } \frac{1}{3} \leq x \leq 1\end{cases}
$$

It satisfies all requirements $1^{\prime}, 2-4,5^{\prime}, 6,7,8^{\prime}, 10,11$, and $12^{\prime}$, but it clearly violates Property 9. The corresponding family of functions is shown in Fig. $6 \mathrm{~b}$. Note that the functions are not strictly convex: In the squares $[0,1 / 3]^{2}$ and $[1 / 3,1]^{2}$ they are straight lines.

3. Here is an uncountably infinite set of families of functions satisfying the requirements $1^{\prime}, 2-4,5^{\prime}, 6,7,8^{\prime}, 9-11$, and $12^{\prime}$. We give only $h, C$, and $\varphi^{-1}$ explicitly. The function

$$
h(z)= \begin{cases}1-\alpha-z & \text { for } z \geq 1 \\ -\alpha z & \text { for } 0 \leq z \leq 1 \\ \alpha z & \text { for }-1 \leq z \leq 0 \\ 1-\alpha+z & \text { for } z \leq-1\end{cases}
$$

is again even and concave (but not strictly concave) for all $0 \leq \alpha \leq 1$ (an uncountably infinite set). We have for $\alpha \neq 0$

$$
\begin{gathered}
C=C_{\alpha}=2\left(\alpha^{-1}+e^{-\alpha}\left(1-\alpha^{-1}\right)\right), \\
\varphi^{-1}(s)= \begin{cases}\frac{1}{C_{\alpha}} e^{1-\alpha+s} & \text { for } s \leq-1, \\
\left.\frac{1}{C_{\alpha}}\left(\left(e^{\alpha s}-e^{-\alpha}\right) / \alpha+e^{-\alpha}\right)\right) & \text { for }-1 \leq s \leq 0, \\
\left.1-\frac{1}{C_{\alpha}}\left(\left(e^{-\alpha s}-e^{-\alpha}\right) / \alpha+e^{-\alpha}\right)\right) & \text { for } 0 \leq s \leq 1, \\
1-\frac{1}{C_{\alpha}} e^{1-\alpha-s} & \text { for } s \geq 1 .\end{cases}
\end{gathered}
$$

The functions $f^{t}$ may be calculated from this by (5). For $\alpha=0.9$ and $\alpha=-1$, these families of functions are shown in Fig. 6c-d. Figure $6 \mathrm{~d}$ illustrates a case where the convexity property does not hold.

4. For Y.-K. Ng's example (21) in [12], we get

$$
h(z)=z-2 \ln \left(1+e^{z}\right) .
$$


This too is even:

$$
h(-z)=-z-2 \ln \left(1+e^{-z}\right)=-z-2 \ln \left(e^{z}+1\right)+2 \ln e^{z}=z-2 \ln \left(1+e^{z}\right)=h(z)
$$

and strictly concave: $h^{\prime \prime}(z)=-2 e^{z} /\left(1+e^{z}\right)^{2}<0$. Let us check the representation in Theorem 5:

$$
\begin{gathered}
C=\int_{-\infty}^{\infty} e^{h(z)} d z=\int_{-\infty}^{\infty} e^{z-2 \ln \left(1+e^{z}\right)} d z=\int_{-\infty}^{\infty} \frac{e^{z}}{\left(1+e^{z}\right)^{2}} d z=-\left.\frac{1}{e^{z}+1}\right|_{z=-\infty} ^{z=\infty}=1, \\
\varphi^{-1}(s)=\frac{1}{C} \int_{-\infty}^{s} e^{h(z)} d z=-\left.\frac{1}{e^{z}+1}\right|_{z=-\infty} ^{z=s}=-\frac{1}{e^{s}+1}+1=\frac{e^{s}}{1+e^{s}}=\frac{1}{1+e^{-s}},
\end{gathered}
$$

as in Sec. 5. This family has been shown in Fig. 3 .

7. Discussion: interpretation of the results. We saw that $\Phi: \mathbb{R} \rightarrow] 0,1[$ is continuous and strictly increasing; furthermore,

$$
\lim _{s \rightarrow-\infty} \Phi(s)=0, \quad \lim _{s \rightarrow \infty} \Phi(s)=1 .
$$

So $\Phi$ is the probability distribution function of a random variable $S$ :

$$
\Phi(s)=\operatorname{Prob}[S \leq s]
$$

and $s=\Phi^{-1}(x)=\varphi(x)$ is the $x$-quantile of $S$, i.e., $s$ is the threshold value for probability $x$ in the sense that the probability that $S$ does not exceed $s$ is $x$. (We could also consider $\Phi$ as a sufficiently smooth approximation of an empirical distribution function of some quantity $S$, replacing the term "probability" by "percentage".) This gives a natural interpretation of (6) and (7), which can now be written as

$$
\Phi^{-1}\left(y_{2}\right)-\Phi^{-1}\left(y_{1}\right)=\Phi^{-1}\left(x_{2}\right)-\Phi^{-1}\left(x_{1}\right)
$$

The right-hand side is the amount by which the threshold must be moved to change the probability from $x_{1}$ to $x_{2}$. By Theorem 1 and, in particular, (7) and (29), the change of probability from $y_{1}$ to $y_{2}$ is "equivalent" to the change from $x_{1}$ to $x_{2}$ if it corresponds to the same difference in threshold values.

The reader may wish to reexamine the "reasonable properties" in [12] and in the present paper in view of this interpretation. They will indeed turn out to be quite natural. All that is needed concerning the random variable $S$ is that it should be continuous and its support should be all of $\mathbb{R}$ in order that $\varphi(x)=\Phi^{-1}(x)$ can be defined for every $x \in] 0,1\left[\right.$ and (29) can be solved uniquely for $y_{2}$ whatever $x_{1}, x_{2}$, and $y_{1}$ are. It is not completely obvious, however, why $S$ should have a positive probability density for the whole set $\mathbb{R}$. This is in fact mainly a consequence of the innocuous-looking property 6 . As mentioned there, it implies that it is not possible to change the probability or proportion $y$ from any value $y_{1}<1$ to $y_{2}=1$ in a manner equivalent to a change between two values $x_{1}$ and $x_{2}$ strictly between 0 and 1 . While this may be reasonable in certain circumstances it can definitely not be taken for granted. One may imagine an alternative 
scenario where the change of variable $y$ from $y_{1}<1$ to $y_{2}=1$ is equivalent to a change from $\left.x_{1} \in\right] 0,1\left[\right.$ to an $\left.x_{2} \in\right] 0,1$. No change of $y$ would then match a further change of $x$ beyond $x_{2}$. (This is apparently the conclusion of the argument in the Newsweek article quoted at the beginning of Sec. 1, which, in such a setting, does not look so absurd after all.) Thus we would have given up Property 1, which says that a matching probability $y_{2}$ exists for all values of $x_{1}, x_{2}$, and $y_{1}$. If, on the other hand, we want to keep Property 1 we might say that any change from $x_{1}$ to a value $x_{2}^{\prime}$ beyond $x_{2}$ is equivalent to the change from $y_{1}$ to 1 . But then there would not exist a unique $x_{2}^{\prime}$ matching the change from $y_{1}$ to 1 , contradicting uniqueness (Property 2).

In the interpretation of $\Phi$ as the distribution of a random variable, Property 9 describes a symmetric distribution, since then the probability density function $\Phi^{\prime}$ is symmetric; cf. the discussion after Theorem 5. Furthermore, the convexity property $12^{\prime}$ means exactly that the density function is log-concave. Indeed, as we have seen in the proof of Theorem 4, Property $12^{\prime}$ means that the function $k$ defined by $(23)$, that is, $k(s)=\ln \Phi^{\prime}(s)$, is concave.

As mentioned before, in Example 1 of Sec. 6,

$$
\Phi(s)=\frac{1}{\sqrt{2 \pi}} \int_{-\infty}^{s} e^{-z^{2} / 2} d z
$$

is the normal (Gaussian) probability distribution with mean 0 and variance 1. For many problems this is clearly the most appropriate basis to find equivalent changes in probabilities by use of formula (29).

The problem of comparing changes in probabilities relates to difference measurement, which goes back at least to O. Hölder in 1901 [8, Sec. 2]. He derived (6) and (7) from other assumptions $[8, \S 23]$; see also [9, Theorem 4.2].

Acknowledgments. The first author is grateful for the kind hospitality of the Institut für Mathematik der Universität Graz and for support from the Natural Sciences and Engineering Research Council of Canada during the writing of this paper. The authors thank PL. Kannappan for suggesting a rephrasing of Property $1^{\prime}$.

\section{REFERENCES}

[1] J. Aczél, Quasigroups, nets, and nomograms, Adv. Math. 1, 383450 (1965)

[2] J. Aczél, Funktionskomposition, Iterationsgruppen und Geuebe, Arch. Math. (Basel) 17, 469-475 (1966)

[3] G. Blanton, Smoothness in disjoint groups of real functions under composition, Aequationes Math. 35, 1-16 (1988)

[4] G. Blanton and J. A. Baker, Iteration groups generated by $C^{n}$ functions, Arch. Math. (Brno) 18, $121-128(1982)$

[5] W. Blaschke and G. Bol, Geometrie der Gewebe, Springer, Berlin. 1938

[6] R. H. Bruck, A Survey of Binary Systems, Springer, Berlin, Heidelberg, New York, 1971

[7] R. L. Devaney, Chaos, Fractals, and Dynamics: Computer Experiments in Mathematics, AddisonWesley, Menlo Park, California, 1990

[8] O. Hölder, Die Axiome der Quantität und die Lehre vom Maß. Berichte über die Verhandlungen der königlich sächsischen Gesellschaft der Wissenschaften zu Leipzig, mathematisch-physische Classe 53, 1-64 (1901)

[9] D. H. Krantz, R. D. Luce, P. Suppes, and A. Tversky, Foundations of Measurement, vol. 1: Additive and Polynomial Representations, Academic Press, New York, London, 1971 
[10] M. Kuczma, An Introduction to the Theory of Functional Equations and Inequalities, PWNUniwersytet Śląski, Warszawa, Kraków, Katowice, 1985

[11] M. A. McKiernan, On iteration groups and related functional equations, Aequationes Math. 31, 37-46 (1986)

[12] Yew-Kwang Ng, Equivalent changes/differences for percentages and probabilities, Quart. Appl. Math. 49, 289-301 (1991)

[13] K. S. Sibirski, Introduction to Topological Dynamics, Noordhoff, Leyden, 1975

[14] M. C. Zdun, Continuous and Differentiable Iteration Semigroups, Uniwersytet Śląski, Katowice, 1979 